\title{
Acute Viral Rhinitis
}

\author{
Tolgahan Çatl1, Huntürk Atilla, and Eva Kathryn Miller
}

\subsection{Introduction}

Rhinitis refers to any kind of inflammatory condition of the nasal mucosal linings. Generally, acute rhinitis is associated with environmental allergies or respiratory viral infections. Viral microbes with numerous types and subtypes can infect the respiratory epithelium of the nasal cavity in a repetitive fashion throughout the year, or during a specific period of time such as winter or fall.

Among all forms of inflammatory diseases of the nasal mucosa, acute viral rhinitis (AVR) has unique epidemiological, clinical, and therapeutic characteristics. As the most prevalent type of rhinitis, AVR is also the most common form of any infectious disease of the human body [1]. Although it is almost always self-limiting, in rare circumstances disease might progress and the clinical scenario could become complicated. Common complaints and physical findings related to AVR are similar to those seen with other types of rhinitis such as allergic, hormonal, senile, or drug induced [2]. The clinician must interpret these symptoms and findings in the context of other parameters such as "duration, environmental factors, and patient characteristics" to establish an accurate diagnose and appropriate therapeutic management. In this chapter, we aim to discuss the epidemiology, pathogenesis, clinical findings, differential diagnosis, and therapeutic management of AVR in light of the recent literature knowledge. It is our hope that this chapter may aid medical professionals who encounter AVR in daily practice.

T. Çatlı $(\bowtie)$

Department of Otorhinolaryngology, University of Health Sciences, Bozyaka Training and Research Hospital, İzmir, Turkey

H. Atilla

Department of Otorhinolaryngology, University of Health Sciences, Yenimahalle Training and Research Hospital, Ankara, Turkey

E. K. Miller

Department of Pediatrics, Vanderbilt University School of

Medicine, Nashville, TN, USA

e-mail: eva.k.miller@vanderbilt.edu

\subsection{Epidemiology}

AVR is the most common form of human respiratory infectious diseases, and the predominant inciting pathogens are composed of numerous types and sub types in nature. Acute infection of the upper airways is usually viral, rather than bacterial. Human rhinoviruses (HRV) are responsible for up to half of the cases of upper respiratory infections (URI) or "colds" [3]. Other viruses such as coronaviruses, adenoviruses, respiratory syncytial virus (RSV), influenza viruses, and parainfluenza viruses account for a relatively minor proportion of viral colds. These viral agents infect the nasal respiratory epithelium and also other components of the upper/lower respiratory tract after inoculation through respiratory droplets of an infected person. Although oral inoculation might be an alternative source of viral transfer, the risk is relatively low. Risk of experiencing AVR in a single year is much more probable for children than adults. While children experience 8-12 colds a year, adults usually experience 2-3 colds per year [4]. Rhinoviruses can be cultured by a nasopharygeal swab for up to 3 weeks after inoculation [5], and even longer in the immune compromised host. Usually, the inflammation is controlled by the host immune response and almost always disease is self-limited. However in some circumstances, the natural course of the disease may be altered and complications may arise such as bacterial sinusitis or otitis, asthma exacerbation, pneumonia, chronic post-viral cough, and cavernous venous thrombosis.

\subsection{Pathogenesis}

Nasal respiratory epithelium has four types of cells: basal, goblet, ciliated, and un-ciliated columnar cells, which are separated from the lamina propria by a basal membrane [6]. Although the nasal mucosa is in persistent and repetitive contact with the outer world and suffers various sources of injuries such as infectious and physical, the mucosa is armed with various mechanisms for epithelial repair and remodeling. 
The usual suspect triggers of AVR are the most common respiratory viruses including human rhinoviruses, RSV, influenza viruses, parainfluenza viruses, and adenoviruses [7]. These viruses infect the nasal epithelial cells, damage tight junctions, disrupt membranes, and induce cell death. The epithelial infection process begins with viral entry into the nasal cell via receptors. The identified receptors for rhinoviruses, the most common cause of AVR, include intercellular adhesion molecule-1 (ICAM-1) and Toll-like receptor 3 (TLR3) [8, 9]. Viral endocytosis is the next step after receptor binding and is followed by expression and duplication of the viral genetic material within a couple of hours after the initial viral and human cell interaction. Rhinovirus-infected cells upregulate some integral molecules such as interleukin$1 \mathrm{~b}$ (IL-1b) and nuclear factor (NF)-kB and these molecules upregulate ICAM-1, enhancing infectivity and promoting inflammatory infiltration [10]. After invasion, the virus begins to dominate host cell metabolism in order to replicate itself, and this usually results in host cell destruction. Cell death after invasion and/or apoptosis may limit viral replication by augmenting antigen presentation and host immune response. Viral inflammation "triggered by the infected cells and augmented by the host immune system" is composed of an activated cascade of numerous biological pathways and results in elimination of the offending agent. This inflammation is also the source of various clinical symptoms experienced during the course of an AVR, due to injury of the nasal mucosal linings.

\subsection{Clinical Findings}

During the inflammation of the nasal mucosa, particularly in the first days of clinical rhinitis symptoms, cellular infiltration of the nasal cavity predominately by the polymorphonuclear leukocytes is the characteristic histopathological finding. These ultrastructural events are usually reflected as the typical symptoms and physical findings of the AVR such as "excessive mucus production and rhinorrhea, nasal blockage, sneezing, watery eyes, and some degree of nasal and ocular pruritus" [11]. This symptom and sign complex is defined as "rhinitis symptom and sign complex (rSSC)" by Doyle et al. [12]. Although many studies in adults experimentally exposed to viruses (HRV, RSV, etc.) showed that even viral replication and shedding is evident, these symptoms and signs are observed in only $50 \%$ of the volunteers [13]. If rSSC is present, it is typically delayed relative to viral exposure and this time period differs between virus types. This delay is about $48 \mathrm{~h}$ for rhinoviruses and about 7 days for RSV [14]. However, the resolution period is usually similar for all types of viruses and typically takes 4-10 days. With these rSSC, in most of the cases selfdiagnosis is not unusual. However, if the symptoms are of longer duration and/or with elevated body temperature, chills and/or body aches, a "flu" diagnosis is probable and clinician must be aware of the possible serious morbidities.

The underlying mechanism of the associated symptoms and physical findings of AVR is basically triggered by the inflammatory cascade of the immune system [15]. For example, the lowered activation threshold of the nasal epithelial neural network that is affected by the inflammatory molecules triggers the sneeze reflex. In a similar manner, nasopharyngeal sensitization occurs and coughing is triggered to facilitate expectoration. Concurrently, nasal epithelial intercellular tight junction remodeling and mucin production allow for the clearance of nasal mucosal surface and also produce a functional barrier from the outer environment. Altered nasal vascular resistance, mainly driven by venous pooling, instigates the increased nasal airway resistance and decreased nasal airway patency by mucosal swelling and excessive mucus secretions.

In daily practice, patients with rSSC almost always present to medical professionals (rhinologists, pulmonologists, family physicians, etc.) with a self-diagnosis, but physicians must acquire a detailed medical history and perform meticulous physical examination for a precise diagnosis and adequate treatment. The first step is taking a detailed history, where the patient is asked about any chief complaints and timing of symptoms, duration, and severity. In a case of AVR, chief symptoms to consider include "excessive mucus production and rhinorrhea, nasal blockage, sneezing, watery eyes, and some degree of nasal and ocular pruritus," as well as "chills, muscle aches, etc." In AVR, these symptoms have an acute beginning and typically resolve after 7 days of a self-limiting course. In an uncomplicated circumstance, blood or other laboratory testing is not necessary and history and physical findings are adequate to make a diagnosis.

On physical examination, the patient's general appearance is very typical with a pale face, watery nose/eyes and reddish nose tip. Detailed upper aero-digestive tract examination reveals "edematous, pale, and watery inferior turbinates bilaterally with a blocked nasal passage." Physicians should assess these anatomic changes when considering this and other possible rhinitis etiologies. Although the main tool for differentiation of important rhinitis etiologies is taking a detailed medical history (including family history); physical examination and laboratory testing are also other important complementary tools. In a patient with early acute rSSC, generalized symptoms and occasional fever are not uncommon; and physicians need to detail the history of the patient to differentiate other etiologies that do not present with generalized symptom or fever. Physical assessment must include systems other than the nasal cavity such as ocular, pulmonary, or systemic findings that may provide some important clues about the nature of the rhinitis. In a patient with rhinitis, accompanying conjunctivitis may be a component of AVR or allergic rhinitis. Cervical lymphadenopathy is also another physical finding commonly associated with AVR and must be considered during the evaluation. 
Table 23.1 Differential diagnosis of rhinitis

\begin{tabular}{|c|c|c|}
\hline Allergic & Non-allergic & $\begin{array}{l}\text { Others that may mimic } \\
\text { symptoms of rhinitis }\end{array}$ \\
\hline $\begin{aligned} &- \text { Perennial/ } \\
& \text { seasonal } \\
& \text { rhinitis } \\
&- \text { Occupational } \\
& \text { rhinitis } \\
& \text { (allergen) }\end{aligned}$ & $\begin{aligned}- & \text { Infectious rhinitis } \\
- & \text { Atrophic rhinitis } \\
- & \text { Drug-induced } \\
& \text { rhinitis } \\
- & \text { Gustatory rhinitis } \\
- & \text { Non-allergic } \\
& \text { rhinitis with } \\
& \text { eosinophilia } \\
& \text { syndrome } \\
& \text { (NARES) } \\
- & \text { Reflux-induced } \\
& \text { rhinitis }\end{aligned}$ & $\begin{array}{ll}\text { - } & \text { Cerebrospinal fluid } \\
\text { rhinorrhea } \\
\text { - } & \text { Inflammatory/ } \\
\text { immunologic } \\
\text { conditions } \\
\text { (Sarcoidosis, } \\
\text { Wegener's } \\
\text { granulomatosis, etc.) } \\
-\quad \text { Foreign bodies } \\
-\quad \text { Nasal tumors } \\
- & \text { Choanal atresia }\end{array}$ \\
\hline
\end{tabular}

\subsection{Differential Diagnosis}

Clinicians must prepare an algorithm in the evaluation of a patient with rhinitis, because some presentations of rhinitis could be eliminated after certain basic questions such as duration of rSSC, accompanying symptoms, and viral history. In a patient with acute symptoms (1 week or less) without a viral exposure history, acute exacerbation of allergic rhinitis is possible and clinicians must detail the medical and family histories to determine if viral pathogenesis is likely or not. Another clinical entity seen in children with an acute rSSC (especially with unilateral symptoms) is a foreign body in the nasal cavity, particularly when there is malodorous discharge. In cases involving longer duration of symptoms, other chronic pathologies such as seasonal/perennial allergic rhinitis or non-allergic rhinitis should be considered. These rhinitis etiologies are summarized in Table 23.1, and also may be categorized by acute or chronic rSSC.

\subsection{Treatment}

For a disease such as AVR which is usually self-diagnosed, limited to a short course and self-treated in a non-professional setting, it is difficult to encourage every patient to use antiviral drugs or even seek medical care. However, in placebocontrolled studies comparing effective antiviral drugs (interferon-alpha [IFN-alpha], Zanamivir, etc.) with placebo, there is consistently earlier resolution of symptoms and signs by about 1 day for both rhinoviruses and influenza [16, 17], in the anti-viral arms. Antiviral agents primarily decrease viral shedding, but other steps of viral infection may be targeted [18]. In usual clinical practice, AVR receives very traditional treatment strategies such as "hydration with plenty of fluids, herbal and nutritional supplements (vitamin C, selenium, zinc, etc.), homemade soups, honey, and multiingredient (antihistamine, oral/topical decongestant, antiinflammatory, etc.) over-the-counter (OTC) drugs." These regimens are reasonable because the basic underlying mechanisms of rSSC are targeted with anti-inflammatory molecules and hydration. Bed rest, activity restriction, and cigarette cessation also offer some degree of symptom relief. Antibiotics are not necessary unless secondary bacterial infection occurs during the viral infection. In the case of suspected bacterial super infection (acute bacterial rhinosinusitis, bacterial lower respiratory tract infection, etc.), these are usually treated as classic bacterial infections for 10-14 days with antibacterial prescriptions. Emerging data suggests that at least 5 days of antibiotic therapy for community-acquired pneumonia may be sufficient, monitored with serial physical assessments. Vaccination is another optimistic strategy in the prevention of AVR by creating an immune memory response to the particular virus subtype [19]. However, the large number of virus subtypes and yearly changing characteristics may dampen the enthusiasm for broad implementation. Application of such a proposed vaccine should be first limited to the "at risk" population such as "young, elderly, and immunocompromised" patients. Concerning transmission routes of these viruses, education strategies of the population about viral dissemination and hand-washing seem to be the best logical option in the fight of our kind against these micro-enemies.

\subsection{Summary}

As one of the most common infectious diseases of human kind, AVR is a familiar, though sometimes insidious phenomenon and should come to the forefront of each clinician's mind when assessing rhinitis in the hopes of preventing possible complications. Human rhinovirus is the most common pathogen responsible for almost $50 \%$ of the cases. rSSC is defined with "excessive mucus production and rhinorrhea, nasal blockage, sneezing, watery eyes, and some degree of nasal and ocular pruritus." There is no standard laboratory or radiological assessment tool for AVR, but if the clinical course becomes complicated, complementary studies should be performed. Particularly in a susceptible individual, AVR might develop complications such as bacterial super infections of the upper or lower airways and thus may further have more serious morbidities and even mortal consequences. In the treatment of an AVR, some palliative strategies such as "bed rest, activity restriction, cigarette cessation, oral and/or parenteral hydration with anti-inflammatory drugs" can be the initial approach although some anti-viral molecules (INF- $\alpha$, Zanamivir, etc.) are shown to be effective in AVR treatment by shortening the duration of rSSC. This is neither a first-line therapy nor is it widespread in practice for the management of a cold. Vaccination is theoretically a good option for the prevention of any viral disease; however, the large number of virus subtypes and yearly changing charac- 
teristics dampen our enthusiasm for their usage. Finally, the education strategies of the population about viral dissemination and cold transmission, including hand-washing/hygiene techniques and prevention of spread, seem to be the best and most logical option in the fight of our kind against these micro enemies.

\section{References}

1. Slavin RG, Spector SL, Bernstein IL, et al. The diagnosis and management of sinusitis: a practice parameter update. J Allergy Clin Immunol. 2005;116:S13-47.

2. Yan Y, Gordon WM, Wang DY. Nasal epithelial repair and remodeling in physical injury, infection, and inflammatory diseases. Curr Opin Otolaryngol Head Neck Surg. 2013;21(3):263-70.

3. Winther B, Gwaltney JM Jr, Mygind N, et al. Viral-induced rhinitis. Am J Rhinol. 1998;12(1):17-20.

4. Dingle JH, Badger GF, Jordan WS Jr. IIIness in the home. A study of 25,000 illnesses in a group of Cleveland families. Cleveland: The Press of Western Reserve University; 1964.

5. Winther B, Gwaltney JM Jr, Mygind N, et al. Sites of rhinovirus recovery after point-inoculation of the upper airway. JAMA. 1986;256:1763-7.

6. Watelet JB, Van Zele T, Gjomarkaj M, et al. Tissue remodelling in upper airways: where is the link with lower airway remodelling? Allergy. 2006;61:1249-58.

7. Fokkens WJ, Lund VJ, Mullol J, et al. European position paper on rhinosinusitisand nasal polyps. Rhinol Suppl. 2012;23:1-298.
8. Papi A, Johnston SL. Rhinovirus infection induces expression of its own receptor intercellular adhesion molecule 1 (ICAM-1) via increased NF-kappaB-mediated transcription. J Biol Chem. 1999;274:9707-20.

9. Lewis TC, Henderson TA, Carpenter AR, et al. Nasal cytokine responses to natural colds in asthmatic children. Clin Exp Allergy. 2012;42:1734-44.

10. Van Kempen M, Bachert C, Van Cauwenberge P. An update on the patho-physiology of rhinovirus upper respiratory tract infections. Rhinology. 1999;37:97-103.

11. Quillen DM, Feller DB. Diagnosing rhinitis: allergic vs. nonallergic. Am Fam Physician. 2006;73(9):1583-90.

12. Doyle WJ, Gentile A, Skoner D. Viral and bacterial rhinitis. Clin Allergy Immunol. 2007;19:177-95.

13. Doyle WJ. Illness and otological changes during upper respiratory virus infection. Laryngoscope. 1999;109:324-8.

14. Doyle WJ, Skoner DP, Gentile D. Nasal cytokines as mediators of illness during the common cold. Curr Allergy Asthma Rep. 2005;5(3):173-81.

15. Bende M. Changes in human nasal mucosa during experimental coronavirus common colds. Acta Otolaryngol. 1989;107(3-4):262-9.

16. Hedrick JA. Zanamivir for treatment of symptomatic influenza A and $\mathrm{B}$ infection in children five to twelve years of age: a randomized controlled trial. Pediatr Infect Dis J. 2000;19(5):410-7.

17. Fendrick AM. Viral respiratory infections due to rhinoviruses: current knowledge, new developments. Am J Ther. 2003;10(3):193-202.

18. Doyle WJ. Effect of rimantadine treatment on clinical manifestations and otologic complications in adults experimentally infected with influenza A (H1N1) virus. J Infect Dis. 1998;177(5):1260-5.

19. Kibble A. 18th International Conference on Antiviral Research. Respiratory viruses. Drugs. 2005;8(6):465-6. 